\title{
Subjective Risk Measures: Bayesian Predictive Scenarios Analysis
}

\author{
Tak Kuen Siu and Hailiang Yang \\ Department of Statistics and Actuarial Science \\ The University of Hong Kong \\ Hong Kong
}

\begin{abstract}
In this paper we study methods for measuring risk. First, we introduce a conditional risk measure and point out that it is a coherent risk measure. Using the Bayesian statistical idea a subjective risk measure is defined. In some special cases, closed form expressions for the risk measures can be obtained. The credibility theory can be used to relax the strong assumptions on the model and prior distributions, and to obtain approximated risk measure formulas. Applications in both finance and insurance are discussed.
\end{abstract}

Key Words: Coherent risk measure, subjective risk measure, Bayesian analysis, credibility theory, risk interval, conditional risk measure, scenario analysis, Bühlmann estimators, global investment.

\section{Acknowledgments}

The authors would like to thank the referee for many helpful comments and suggestions. This work was partially supported by a grant from Research Grants Council of HKSAR (Project No. HKU 7168/98H). 


\section{$\S 1$ Introduction}

Risk measurement is one of the most important issues in the financial and insurance industries. In the financial industry, volatility is a commonly used measure of risk and ruin probability has been used by insurance industries for many years. Developed by the commercial bank J.P. Morgan, value at risk (VaR) has become very popular. VaR is an attempt to provide a single number summarizing the total risk in a portfolio of financial assets. For an introduction on VaR, see J.P. Morgan's RiskMetrics - Technical Document, for a survey on this subject see the paper by Duffie and Pan (1997).

In Artzner, Delbaen, Eber and Heath (1998), both market and non-market risks have been studied and a set of four desirable properties for measures of risk are presented and justified. A risk measure which satisfies the four properties is called a coherent risk measure. It has been pointed out in Artzner et al. (1998) that value at risk does not satisfy some of the four properties. Motivated by Artzner et al. paper, Cvitanic and Karatzas (1998) have studied the dynamic measures of risk. Such a risk measure is also discussed by Follmer and Leukert (1998).

Risk can be defined as an exposure to uncertainty, (see Holton (1997)), although different people may have different views on uncertainty. Even the same person looking at the uncertainty from different perspectives may obtain different conclusions. Therefore risk is subjective, however we should include subjective views when we model risk. In this paper, motivated by Holton (1997), we extend the model of Artzner et al. (1998) by including a subjective view in our model.

Our goal is to construct a model for risk measurement which captures both the objective market data and the subjective view of the risk trader. We first define a conditional risk measure and point out that it is a coherent risk measure. Then we define a Bayesian risk measure and obtain its closed form expressions in some special cases.

Using the credibility approach, we relax the stringent conjugate-prior assumptions. The advantage of the credibility approach is that we do not have to impose assumptions on the model and prior distributions, and we are able to obtain an approximated expression for the subjective risk measure. The subjective risk measure can be applied 
to both the financial risk and insurance risk.

Finally, we will give an alternative way to capture the random effect of foreign exchange(FX) rates. A modification of the Bayesian risk measure is also introduced. The central tenet of this paper can be applied to value at risk (VaR). We shall discuss subjective VaR in a forthcoming paper.

\section{$\S 2$ Conditional risk measure}

In this section, we define a risk measure for holding a portfolio over the time interval $[n, n+1]$ given the market information (or data) up to time $n$. We present the risk measure model under the dynamic framework. As more information comes, the risk measure can be updated sequentially. In this way, we can mark to market by adjusting the risk measure in the daily balance sheet.

Let $\Omega$ be the set of all states of nature. $\mathcal{F}$ be a $\sigma$-algebra on $\Omega$. We equip our sample space $(\Omega, \mathcal{F})$ with a filtration $\left\{\mathcal{F}_{n}\right\}$, where $\mathcal{F}_{n} \subseteq \mathcal{F}_{n+1}$ and $\mathcal{F}_{n}$ is a sub- $\sigma$-field of $\mathcal{F}$ for all $n$. Here, $\mathcal{F}_{n}$ represents the market information (or data) up to time $n$. Let $\mathbb{P}$ be a probability measure on $(\Omega, \mathcal{F})$. For our purpose here, we assume that $\mathbb{P}$ is a subjective probability. Let $P$ denote a family of subjective probability measures $\mathbb{P}$ (i.e. the set of all "scenarios").

Let $\Delta X_{i}$ be the change of market value (measured in terms of the domestic currency of the risk trader) of a portfolio over the time interval $[i-1, i]$, where $\Delta X_{i}: \Omega \rightarrow \mathbb{R}$ is a random variable, for $i=1,2, \ldots, n, n+1$. $\mathcal{G}_{n+1}$ denotes the set of all functions $\Delta X_{n+1}: \Omega \rightarrow \mathbb{R}$, (i.e. the set of all risks over the time interval $[n, n+1]$. Note that $\left\{\Delta X_{i}\right\}$ is adapted to $\left\{\mathcal{F}_{i}\right\}$, for $i=1,2, \ldots, n, n+1$.

Then, the conditional risk measure is a function $\rho_{P}\left(\cdot \mid \mathcal{F}_{n}\right): \mathcal{G}_{n+1} \rightarrow \mathbb{R}$ defined as

$$
\rho_{P}\left(\Delta X_{n+1} \mid \mathcal{F}_{n}\right)=\sup \left\{-E_{\mathbb{P}}\left(\frac{\Delta X_{n+1}}{r_{n}} \mid \mathcal{F}_{n}\right) \mid \mathbb{P} \in P\right\} \quad \text { for } \quad \Delta X_{n+1} \in \mathcal{G}_{n+1}
$$

Here, $r_{n}$ is the return of a dollar invested in a reference instrument over the time interval $[n, n+1] . \rho_{P}\left(\Delta X_{n+1} \mid \mathcal{F}_{n}\right)$ measures the risk of holding a portfolio over the time interval $[n, n+1]$ given the market information up to time $n$. It takes into account the worst "scenario" among all possible "scenarios" given the market information up to time $n$. 


\section{Remarks}

In Wang, Young and Panjer (1997), four axioms were presented to describe the behavior of market insurance prices. Chateauneuf, Kast and Lapied (1996) considered properties of pricing rules in financial markets with frictions. Wang and Young (1998) combined credibility theory with the premium principle proposed in Denneberg (1994) and presented a risk adjusted credibility premium calculation formula. Young (1998) introduced a family of updating rules to update prices in light of newly available information. In Cvitanic and Karatzas (1998), a dynamic measure of risk was proposed to measure the risk of a portfolio containing derivatives. The conditional risk measure in this paper is rather general, and as we point out that it satisfies the four coherence properties given in Artzner et al. (1998), it is a coherent risk measure.

\section{Example}

Assume under $\mathbb{P} \in P, \Delta X_{n+1} \mid \mathcal{F}_{n} \sim N\left(\mu_{n}^{\mathbb{P}}, \sigma_{n}^{\mathbb{P}}\right)$, where $\mu_{n}^{\mathbb{P}}$ and $\sigma_{n}^{\mathbb{P}}$ are $\mathcal{F}_{n}$-measurable random variables, so $\mu_{n}^{\mathbb{P}}$ and $\sigma_{n}^{\mathbb{P}}$ become known at time $n$. The conditional risk measure in this case becomes:

$$
\rho_{P}\left(\Delta X_{n+1} \mid \mathcal{F}_{n}\right)=\sup \left\{-\frac{\mu_{n}^{\mathbb{P}}}{r_{n}} \mid \mathbb{P} \in P\right\}
$$

The following is the definition of the coherent risk measure given in Artzner, et al.(1998).

Definition: A risk measure $\rho$ satisfying the following four properties is called coherent:

(i). Translation invariance: for all $X \in \mathcal{G}$ and all real number $\alpha$, we have $\rho(X+\alpha r)=$ $\rho(X)-\alpha$, where $\mathcal{G}$ is the set of all risk, that is the set of all real-valued functions on $\Omega$.

(ii). Subadditivity: for all $X_{1}$ and $X_{2} \in \mathcal{G}, \rho\left(X_{1}+X_{2}\right) \leq \rho\left(X_{1}\right)+\rho\left(X_{2}\right)$.

(iii). Positive homogeneity: for all $\lambda \geq 0$ and all $X \in \mathcal{G}, \rho(\lambda X)=\lambda \rho(X)$.

(iv). Monotonicity: for all $X$ and $Y \in \mathcal{G}$ with $X \leq Y$, we have $\rho(Y) \leq \rho(X)$.

It is not difficult to see that $\rho_{P_{n}}$ defined above is a coherent risk measure.

\section{$\S 3$ Bayesian risk measure}

In section 2 , we use subjective probability measures to capture the model risk, that is we use the "worst scenarios" to define a conservative risk measure. In this section, we 
construct a risk measure by including subjective views in our model and using Bayesian ideas. Bayesian risk measure is another way to capture the model risk. We will try to do so by using predictive distribution to estimate the true distribution. By introducing the Bayesian statistics to our risk measure model, we can capture both the subjective views of the risk traders and the information provided by market data.

We first give a theoretical framework. Let $\mathcal{T}$ be a index set which represents the set of all subjective views chosen by a trader or a group of traders. For each $v \in \mathcal{T}$, let

$$
\left(\Omega \times E, \mathcal{F} \otimes \mathcal{E}, \Pi^{v}\right)
$$

be a Bayesian experiment, where $(E, \mathcal{E})$ is called the parameter space and $(\Omega, \mathcal{F})$ is called the sample space. The product measure $\Pi^{v}=\mathbb{Q}_{v} \otimes \mathbb{P}^{\mathcal{E}}$, where $\mathbb{Q}_{v}$ and $\mathbb{P}$ are respectively the prior and the predictive probabilities. Note that for each fixed $e \in E$, the probability measure $\mathbb{P}^{e}$ is called the sampling probability. (For details see Florens, Mouchart and Rolin (1990)).

Let

$$
\underline{\Theta}:\left(E, \mathcal{E}, \mathbb{Q}_{v}\right) \rightarrow\left(R^{n}, \mathcal{B}\left(R^{n}\right)\right), \quad v \in \mathcal{T}
$$

be a random vector. The distribution of $\underline{\Theta}$ under $\mathbb{Q}_{v}$ is given by:

$$
\underline{\Theta} \sim \pi_{v}(\underline{\theta})
$$

Let $\Delta X_{i}$ be the change in market value ( measured in terms of the domestic currency of the risk trader ) of a portfolio over the time interval $[i-1, i]$ (i.e. the $i$-th period's net worth of a portfolio), for $i=1,2, \ldots, n . n+1$. The random variable $\underline{\Theta}$ is the risk characteristic of a risk trader. Let the vector $\underline{\Delta x_{n}}=\left(\Delta x_{1}, \ldots, \Delta x_{n}\right)$ which represents the market data up to time $n$.

For fixed $e \in E, \underline{\Theta}(e)=\underline{\theta}(e)$. Under $P_{e}, \Delta X_{i} \mid \underline{\Theta}(e)=\underline{\theta}(e), i=1,2, \ldots, n, n+1$, are conditionally independent and identically distributed with common distribution $F(x \mid \underline{\theta}(e))$ (Note that $F(x \mid \underline{\theta}(e))$ is the sampling distribution). Due to the natures of both financial and insurance data, the assumption that $\Delta X_{i} \mid \underline{\Theta}(e)=\underline{\theta}(e), i=1,2, \ldots, n$, are identically distributed hold only when $n$ is not too large. 
Then, for each $v \in \mathcal{T}$, the posterior density of $\underline{\Theta}$ given $\underline{\Delta x_{n}}$ can be calculated by the Bayes' formula as follows:

$$
\pi_{v}\left(\underline{\theta} \mid \underline{\Delta x_{n}}\right)=C \prod_{i=1}^{n} f\left(\Delta x_{i} \mid \underline{\theta}\right) \pi_{v}(\underline{\theta})
$$

(where $C$ is a normalization constant.)

The predictive distribution of the next period's net worth of the portfolio (i.e. the $(n+1)$-th period's net worth of the portfolio $\Delta X_{n+1}$ given the data $\left.\Delta x_{n}\right)$ can be calculated by following the standard steps (see Klugman et al. (1998)).

$$
F_{\Delta X_{n+1} \mid \underline{\Delta x_{n}}}^{v}(x)=C \int \ldots \int F(x \mid \underline{\theta})\left[\prod_{i=1}^{n} f\left(\Delta x_{i} \mid \underline{\theta}\right)\right] \pi_{v}(\underline{\theta}) d \underline{\theta}
$$

This expression depends on $\pi_{v}(\underline{\theta})$ which is chosen subjectively by the risk trader.

Suppose a group of risk traders $\mathcal{T}$ are involved in choosing the model and prior densities. Without loss of generality, we can assume that each risk trader in the group $\mathcal{T}$ chooses exactly one model density and one prior distribution, such that their predictive distributions are all different from each other. Let $P_{n}$ be a family of all predictive distributions chosen by the risk traders in the group $\mathcal{T}$. Then, we can write $P_{n}$ as $\left\{F_{\Delta X_{n+1} \mid \underline{\Delta x_{n}}}^{v}(x) \mid v \in \mathcal{T}\right\}$, where $F_{\Delta X_{n+1} \mid \underline{\Delta x_{n}}}^{v}(x)$ is chosen by the risk trader $v \in \mathcal{T}$ through the model density and the prior density. Also, $F_{\Delta X_{n+1} \mid \underline{\Delta x_{n}}}^{v}(x)$ includes the objective information through the market data $\Delta x_{n}$ up to time $n$.

Then, we define "the subjective ( Bayesian ) risk measure" of the $(n+1)$-th period's net worth $\Delta X_{n+1}$ given the market data $\underline{\Delta x_{n}}$ as:

$$
\rho_{P_{n}}\left(\Delta X_{n+1} \mid \underline{\Delta x_{n}}\right)=\sup \left\{-E_{F^{v}}\left(\Delta X_{n+1} / r_{n} \mid \underline{\Delta x_{n}}\right) \mid v \in \mathcal{T}\right\}
$$

where $F^{v}=F_{\Delta X_{n+1} \mid \underline{\Delta x_{n}}}^{v}(x)$.

We have pointed out that the same person may also have different views about uncertainty in financial markets. Several "scenarios" may be chosen to evaluate the risk of his/her portfolios. In this case, we interpret $\mathcal{T}$ as the index set of all predictive distributions chosen by this person. A set of "scenarios" can be generated through choosing a set of prior densities. We have the following interpretation of our risk measure $\rho_{P_{n}}$. 
Suppose $C_{n}$ is the cash amount in the investor's account at the current time $n$. If $\rho_{P_{n}}\left(\Delta X_{n+1} \mid \underline{\Delta x_{n}}\right)$ is positive, then $\max \left(\rho_{P_{n}}\left(\Delta X_{n+1} \mid \underline{\Delta x_{n}}\right)-C_{n}, 0\right)$ can be interpreted as the call margin added to the investor's account and invested in a reference instrument with return $r_{n}$ in order to support the maximum expected loss. If $\rho_{P_{n}}\left(\Delta X_{n+1} \mid \underline{\Delta x_{n}}\right)$ is negative, then $\min \left(-\rho_{P_{n}}\left(\Delta X_{n+1} \mid \underline{\Delta x_{n}}\right), C_{n}\right)$ can be interpreted as the cash amount that can be withdrawn from the investor's current account so that it can still support the maximum expected loss in the portfolio during the next period. $\rho_{P_{n}}\left(\Delta X_{n+1} \mid \underline{\Delta x}\right)$ is the most conservative (or risk-averse) measure.

Instead of summarizing the risk of a portfolio by a single number, we introduce a risk interval for risk measurement and impose the risk limits or the call margin limits as follows:

$$
\begin{array}{cc}
{\left[\inf \left\{-E_{F^{v}}\left(\frac{\Delta X_{n+1}}{r_{n}} \mid \underline{\Delta x_{n}}\right) \mid v \in \mathcal{T}\right\},\right.} & \left.\sup \left\{-E_{F^{v}}\left(\frac{\Delta X_{n+1}}{r_{n}} \mid \underline{\Delta x_{n}}\right) \mid v \in \mathcal{T}\right\}\right] \\
\uparrow & \text { upper limit }=b_{n}=\rho_{P_{n}}\left(\Delta X_{n+1} \mid \underline{\Delta x_{n}}\right)
\end{array}
$$

If both $a_{n}$ and $b_{n}$ are negative, then $\min \left(-a_{n}, C_{n}\right)$ is the largest amount that can be withdrawn from the investor's account and $\min \left(-b_{n}, C_{n}\right)$ the smallest, so that the cash amount in the account will still be sufficient to cover the expected loss of the portfolio during the next period. Suppose both $a_{n}$ and $b_{n}$ are non-negative. If the margin call is less than $\min \left(a_{n}-C_{n}, 0\right)$, then it is unlikely that the account balance will be sufficient to support the expected loss in the next period, while if the margin call is greater than $\max \left(b_{n}-C_{n}, 0\right)$, then it is considered more than sufficient to cover the maximum expected loss. Each risk trader can decide how much cash will be added to their current account and invested in a reference instrument. The amount of cash should be within the range $\left[\min \left(a_{n}-C_{n}, 0\right), \max \left(b_{n}-C_{n}, 0\right)\right]$. Risk managers can set the margin call requirement for the portfolio based on the range $\left[\min \left(a_{n}-C_{n}, 0\right), \max \left(b_{n}-C_{n}, 0\right)\right]$, the financial situations of their clients and themself, and the competitive condition of the market.

\section{Remarks:}

(1) $r_{n}$ is close to 1 if the length of the period is short. The length of the time period is 
usually one day and $r_{n}$ is considered to be the return rate of an overnight riskless instrument.

(2) If investors think that central banks will intervene in the market and that this may affect an extreme movement of the market value of their portfolios, then they can set the prior mean of $\Theta$ as an extreme value in order to capture the effect of central bank intervention. For more detailed treatment and application of extreme value theory, see Embrechts et al.(1997).

Before ending this section, we would like to point out that the subjective (Bayesian) risk measure satisfies the properties of the coherent measure of risk. However, the properties of subadditivity need some restrictions. Now, we would like to briefly state and interpret these properties.

(1) Translation invariance:

If we add $\alpha_{n}$ amount of capital into the current account/portfolio and invest it in a riskless instrument with return rate $r_{n}$, then we reduce the risk of the portfolio at the next period by the same amount $\alpha_{n}$. If we let $\alpha_{n}$ be $\rho_{P_{n}}\left(\Delta X_{n+1} \mid \underline{\Delta} x_{n}\right)$, then the property of translation invariance becomes " $\rho_{P_{n}}\left(\Delta X_{n+1}+r_{n} \rho_{P_{n}}\left(\Delta X_{n+1} \mid \underline{\Delta x_{n}}\right) \mid \underline{\Delta x_{n}}\right)=$ $0 "$. This means that if we add $\max \left(\rho_{P_{n}}\left(\Delta X_{n+1} \mid \underline{\Delta x_{n}}\right)-C_{n}, 0\right)$ amount of cash to the investor's current account/portfolio and invest it in a riskless instrument at time $n$ with return rate $r_{n}$, then the risk measure for the portfolio at the $(n+1)$-th period is non-negative.

(2) Positive Homogeneity:

If the next period's net worth is multiplied by a factor $\lambda$, then its' Bayesian risk measure is multiplied by the same factor $\lambda$. This implies that we can use different units or currencies to define our risk measure.

(3) Monotonicity:

If there are two portfolios $X$ and $Y$ which satisfy the condition of $\Delta X_{n+1} \leq$ $\Delta Y_{n+1}$, then from the definition of our subjective (Bayesian) risk measure, we have $\rho_{P_{n}}\left(\Delta X_{n+1} \mid \underline{\Delta x_{n}}\right) \geq \rho_{P_{n}}\left(\Delta Y_{n+1} \mid \underline{\Delta y_{n}}\right)$, since our risk measure is defined as the (conditional) expectation of portfolio change. 


\section{(4) Subadditivity:}

If $X$ and $Y$ are independent, then the subadditivity property is hold. If the information provided by given $\underline{\Delta x_{n}}$, or $\underline{\Delta y_{n}}$ is same as the information given by $\Delta x_{n}+\Delta y_{n}$, then the subadditivity is also true. In general we cannot say that the subadditivity property hold.

\section{$\S 4$ A special case (normal-normal conjugate prior case)}

In this section, the normality assumption is imposed for both the prior and model densities. Risk traders can choose the prior means and variances based on their subjective views. Also, they can choose the sampling variance based on estimates from market data. A closed form solution is obtained in this case.

\section{Remarks:}

From the central limit theorem, the normality assumption for the market value of the portfolio can still be acceptable, even though the portfolio consists of some non-linear financial instruments (e.g. stock options, bond options, FX swaps, etc). Although the density function of the domestic price for a foreign security may not be normal, the normality assumption for the domestic price of the whole portfolio is acceptable, provided that the portfolio consists of a sufficiently large number of securities. It is true that empirical studies do not support the normality assumption due to the number of securities in the portfolio usually not being large enough, and the independent assumption of the central limit theorem may not hold. However, the normality assumption as an approximation property is still acceptable in many cases.

Note that $\mathcal{T}$ is a finite set in reality. Suppose $\mathcal{T}=\left\{T_{1}, T_{2}, \ldots, T_{m}\right\}, m$ is a finite

positive integer. A model for the trader $T_{j}$ (for $j=1,2, \ldots, m$ ) is given by:

$$
\begin{gathered}
\Delta X_{i} \mid \Theta=\theta \stackrel{\text { i.i.d. }}{\sim} N\left(\theta, v_{j}\right) \text { for } i=1,2, \ldots, n, n+1 \\
\Theta \sim N\left(\mu_{j}, a_{j}\right)
\end{gathered}
$$

We first assume $v_{j}$ is known for simplicity (this assumption can be released by introducing a prior for $v_{j}$; see the later part of this section). Then, after some calculations, 
we have that the predictive distribution of the trader $T_{j}$ denoted by $F_{\Delta X_{n+1} \mid \underline{\Delta x_{n}}}^{T_{j}}(x)$ is:

$$
N\left(\frac{\frac{n \overline{\Delta x_{n}}}{v_{j}}+\frac{\mu_{j}}{a_{j}}}{\frac{n}{v_{j}}+\frac{1}{a_{j}}},\left(\frac{n}{v_{j}}+\frac{1}{a_{j}}\right)^{-1}+v_{j}\right)
$$

Therefore,

$$
\begin{aligned}
E_{F^{T_{j}}}\left(\Delta X_{n+1} / r_{n} \mid \underline{\Delta x_{n}}\right) & =\frac{1}{r_{n}} E_{F^{T_{j}}}\left(\Delta X_{n+1} \mid \underline{\Delta x_{n}}\right) \\
& =\frac{1}{r_{n}}\left[\left(\frac{n}{v_{j}}+\frac{1}{a_{j}}\right)^{-1} \frac{n}{v_{j}} \overline{\Delta x_{n}}+\left(\frac{n}{v_{j}}+\frac{1}{a_{j}}\right)^{-1} \frac{1}{a_{j}} \mu_{j}\right]
\end{aligned}
$$

Hence,

$$
\begin{aligned}
\rho_{P_{n}}\left(\Delta X_{n+1} \mid \underline{\Delta x_{n}}\right)= & \sup \left\{\frac{-1}{r_{n}}\left[\left(\frac{n}{v_{j}}+\frac{1}{a_{j}}\right)^{-1} \frac{n}{v_{j}} \overline{\Delta x_{n}}+\left(\frac{n}{v_{j}}+\frac{1}{a_{j}}\right)^{-1} \frac{1}{a_{j}} \mu_{j}\right] \mid\right. \\
& 1 \leq j \leq m\} .
\end{aligned}
$$

As long as we can find the predictive means for all traders $T_{j} \in \mathcal{T}$, the exact numerical value of $\rho_{P_{n}}\left(\Delta X_{n+1} \mid \underline{\Delta x_{n}}\right)$ can be found. Also, we can define the lower and upper risk limits as follows:

$$
\begin{gathered}
\rho_{P_{n}}^{\inf }\left(\Delta X_{n+1} \mid \underline{\Delta x_{n}}\right)=\inf \left\{-\frac{1}{r_{n}}\left[\left(\frac{n}{v_{j}}+\frac{1}{a_{j}}\right)^{-1} \frac{n}{v_{j}} \overline{\Delta x_{n}}+\left(\frac{n}{v_{j}}+\frac{1}{a_{j}}\right)^{-1} \frac{1}{a_{j}} \mu_{j}\right] \mid j=1,2, \ldots, m\right\} \\
\rho_{P_{n}}^{\sup }\left(\Delta X_{n+1} \mid \underline{\Delta x_{n}}\right)=\rho_{P_{n}}\left(\Delta X_{n+1} \mid \underline{\Delta x_{n}}\right)
\end{gathered}
$$

So, we have a risk interval $\left[\rho_{P_{n}}^{\inf }\left(\Delta X_{n+1} \mid \underline{\Delta x_{n}}\right), \rho_{P_{n}}^{\text {sup }}\left(\Delta X_{n+1} \mid \underline{\Delta x_{n}}\right)\right]$.

Now, we give an extension of the above model by considering the variance of the sampling density as a random variable, and choosing Gamma as its' prior distribution. The model for trader $T_{j}$ (for $j=1,2, \ldots, m$ ), in this case, is given by:

$$
\begin{gathered}
\Delta X_{i} \mid \Theta, \tau \sim N(\Theta, 1 / \tau) \\
\Theta \mid \tau \sim N\left(\mu_{j}, 1 / a_{j} \tau\right), \quad \tau \sim G_{a}\left(\alpha_{j}, \beta_{j}\right)
\end{gathered}
$$

After some calculations, we know that the predictive density of $\Delta X_{n+1} \mid \underline{\Delta x_{n}}$ is a $t$ density with mean

$$
\frac{a_{j}}{a_{j}+n} \mu_{j}+\frac{n}{a_{j}+n} \overline{\Delta X_{n}}=\left(1-z_{j}\right) \mu_{j}+z_{j} \overline{\Delta X_{n}},
$$


where $z_{j}=\frac{n}{a_{j}+n}$.

In order to apply our model, the prior parameters $\mu_{j}, a_{j}, \alpha_{j}, \beta_{j}$ should be chosen by the risk trader $T_{j}$ based on prior information/experience and their subjective view. In practice, it is difficult to set the parameters $\alpha_{j}$ and $\beta_{j}$ based on the information provided by market data. However, the predictive mean does not depend on the parameters $\alpha_{j}$ and $\beta_{j}$, so the risk measure does not depend on them either. We can use $a_{j}$ to indicate the variability of the prior information. If the variability of the prior information is large, then $a_{j}$ should be small and hence $z_{j}$ will be large. This implies that more weight will give to the market data. The risk trader $T_{j}$ will be inclined to use market data to estimate $\Delta X_{n+1}$. In this way, $z_{j}$ can be interpreted as a credibility factor placed on market data. On the other hand, if the risk trader $T_{j}$ has strong confidence in their prior guess, they can let $a_{j}$ be a large value so that they can make the prior variance of $\Theta \mid \tau$ small. Again, we define the risk limits as follows:

$$
\rho_{P_{n}}^{\sup }\left(\Delta X_{n+1} \mid \underline{\Delta x_{n}}\right)=\sup \left\{-\frac{1}{r_{n}}\left[\left(1-z_{j}\right) \mu_{j}+z_{j} \overline{\Delta x_{n}}\right] \mid j=1,2, \ldots, m\right\}
$$

and

$$
\rho_{P_{n}}^{\inf }\left(\Delta X_{n+1} \mid \underline{\Delta x_{n}}\right)=\inf \left\{-\frac{1}{r_{n}}\left[\left(1-z_{j}\right) \mu_{j}+z_{j} \overline{\Delta x_{n}}\right] \mid j=1,2, \ldots, m\right\}
$$

where $z_{j}=\frac{a_{j}}{n+a_{j}} \cdot\left[\rho_{P_{n}}^{\inf }\left(\Delta X_{n+1} \mid \underline{\Delta x_{n}}\right), \rho_{P_{n}}^{\text {sup }}\left(\Delta X_{n+1} \mid \underline{\Delta x_{n}}\right)\right]$ is the risk interval.

\section{$\S 5$ Credibility theory approach}

Sometimes, it is difficult to find the predictive mean $E_{F^{T_{j}}}\left(\Delta X_{n+1} \mid \underline{\Delta x_{n}}\right)$, especially when the predictive distribution $F^{T_{j}}$ is not known (i.e. not a conjugate-prior case). In this section, we employ the "Bühlmann least square model" in credibility theory to approximate $E_{F^{T_{j}}}\left(\Delta X_{n+1} \mid \underline{\Delta x_{n}}\right)$ as a linear combination of the past market data $\underline{\Delta x_{n}}$ and prior means.

\section{Assumptions:}

(1) $E\left(\Delta X_{i} \mid \Theta\right)=\Theta$ and $\operatorname{Var}\left(\Delta X_{i} \mid \Theta\right)=v_{j n}, i=1,2, \ldots, n, n+1 . v_{j n}$ can be estimated by the trader $T_{j}$ from the mean and volatility (or variation) of the market data up to time $n$. 
(2) $\Delta X_{i} \mid \Theta, i=1,2, \ldots, n, n+1$, are assumed to be conditionally independent with common mean $\Theta$ and variance $v_{j n}$.

(3) $E(\Theta)=\mu_{j}$ and $\operatorname{Var}(\Theta)=a_{j}$.

Here we do not assume any specific form of probability distributions for $\Theta$ and $\Delta X_{i} \mid \Theta$. So, $F_{\Delta X_{n+1} \mid \underline{\Delta x_{n}}}^{T_{j}}(x)$ is not known. Hence, the closed form expression of $E_{F^{T_{j}}}\left(\Delta X_{n+1} \mid \underline{\Delta X_{n}}\right)$ cannot be calculated. We will approximate $E_{F^{T_{j}}}\left(\Delta X_{n+1} \mid \underline{\Delta X_{n}}\right)$ by the linear combination $\alpha_{0}^{T_{j}}+\sum_{k=1}^{n} \alpha_{k}^{T_{j}} \Delta X_{k}$ (i.e. we need to determine $\alpha_{k}^{T_{j}}$ for $k=0,1,2, \ldots, n$ ). From standard results, see for example Klugman et al. (1998) or Willmot (1994), we have:

$$
\begin{aligned}
\alpha_{0}^{T_{j}} & =\mu_{j}\left(1-\frac{n a_{j}}{n a_{j}+v_{j n}}\right)=\left(\frac{v_{j n}}{n a_{j}+v_{j n}}\right) \mu_{j} \\
\alpha_{k}^{T_{j}} & =\left(n a_{j}+v_{j n}\right)^{-1} a_{j} \quad \text { for } \quad k=1,2, \ldots, n .
\end{aligned}
$$

So,

$$
\begin{aligned}
E_{F^{T_{j}}}\left(\Delta X_{n+1} \mid \underline{\Delta x_{n}}\right) & \approx \alpha_{0}^{T_{j}}+\sum_{k=1}^{n} \alpha_{k}^{T_{j}} \Delta x_{k} \\
& =\left(\frac{v_{j n}}{n a_{j}+v_{j n}}\right) \mu_{j}+\left(\frac{n a_{j}}{n a_{j}+v_{j n}}\right) \overline{\Delta x_{n}} \\
& =\left(1-z_{j n}\right) \mu_{j}+z_{j n} \overline{\Delta x_{n}}
\end{aligned}
$$

where the credibility factor placed on the market data and chosen by the risk trader $T_{j} \in \mathcal{T}$, which is denoted by $z_{j n}$, equals $\frac{n a_{j}}{n a_{j}+v_{j n}}$.

\section{Remarks:}

(1) In the normal-normal conjugate prior case, approximations to the lower and upper risk limits by using the credibility approach equal the exact lower and upper risk limits. In general, this approximation is the best one in the sense of linear least square estimation.

(2) $\left(1-z_{j n}\right) \mu_{j}+z_{j n} \overline{\Delta x_{n}}$ is also a least square estimator for both $E\left(\Delta X_{n+1} \mid \Theta\right)=\Theta$ and $\Delta X_{n+1}$.

(3) $z_{j n}$ can be updated sequentially by updating the values of $n$ and $v_{j n}$ as information is updated. 
(4) When the variability of the market data up to time $n$ becomes large, $v_{j n}$ becomes large and $z_{j n}$ becomes small. So, we put small weight on the market data.

From $(*)$ we have

$$
E_{F^{T_{j}}}\left(\frac{\Delta X_{n+1}}{r_{n}} \mid \underline{\Delta x_{n}}\right) \approx \frac{1}{r_{n}}\left[\left(1-z_{j n}\right) \mu_{j}+z_{j n} \overline{\Delta x_{n}}\right]
$$

Now, suppose that $\mathcal{T}=\left\{T_{1}, \ldots, T_{m}\right\}$. Then, we approximate the lower and upper risk limits as follows:

$$
\begin{aligned}
\rho_{P_{n}}^{\inf }\left(\Delta X_{n+1} \mid \underline{\Delta x_{n}}\right) & \approx \inf \left\{-\frac{1}{r_{n}}\left[\left(1-z_{j n}\right) \mu_{j}+z_{j n} \overline{\Delta x_{n}}\right] \mid j=1,2, \ldots, m\right\}=a_{n} \\
\rho_{P_{n}}^{\sup }\left(\Delta X_{n+1} \mid \underline{\Delta x_{n}}\right) & \approx \sup \left\{-\frac{1}{r_{n}}\left[\left(1-z_{j n}\right) \mu_{j}+z_{j n} \overline{\Delta x_{n}}\right] \mid j=1,2, \ldots, m\right\}=b_{n}
\end{aligned}
$$

Therefore, $\left[a_{n}, b_{n}\right]$ is the approximated risk interval. Since we only impose the mean and variance condition for the density function of the change in the market value of a portfolio, the approximated risk measure can be applied to measure the risk of a portfolio in terms of the domestic currency of the risk trader, which consists of both the foreign financial securities and non-linear instruments.

\section{$\S 6$ Generalized Bayesian premium calculation (Bayesian "scenarios" analysis in credibility theory)}

In credibility theory, we are interested in calculating the policy holder's premium of the $(n+1)$-th period given the policy holder's claimed experience during the first $n$ periods. Suppose we have a set of manual rates, each of them corresponds to one type of policy holder. Now, we want to calculate the $(n+1)$-th period's premium of a policy holder based on this set of manual rates and the policy holder's past claim records up to the $n$-th period.

Let $\Delta X_{i}$ be the claim amount of a policy holder during the $i$-th period.

Let $\mathcal{T}$ be the set of manual rates in an insurance company. Assume there are $m$ manual rates in the insurance company. (i.e. $\mathcal{T}=\left\{T_{1}, \ldots, T_{m}\right\}$ ) 
For each manual rate $T_{j} \in \mathcal{T}$, we would like to calculate (if it is a conjugate-prior case )/approximate (if $E_{F^{T_{j}}}\left(\Delta X_{n+1} \mid \underline{\Delta x_{n}}\right)$ is difficult to calculate) $E_{F^{T_{j}}}\left(\Delta X_{n+1} \mid \Delta x_{n}\right)$. As in the previous section, $E_{F^{T_{j}}}\left(\Delta X_{n+1} \mid \Delta x_{n}\right)$ can be approximated by

$$
\alpha_{0}^{T_{j}}+\sum_{k=1}^{n} \alpha_{k}^{T_{j}} \Delta x_{k}
$$

In this way, we get a set of approximations for $E_{F^{T_{j}}}\left(\Delta X_{n+1} \mid \underline{\left.\Delta x_{n}\right)}\right.$ as follows:

$$
\alpha_{0}^{T_{j}}+\sum_{k=1}^{n} \alpha_{k}^{T_{j}} \Delta x_{k}=\left(1-z_{j n}\right) \mu_{j}+z_{j n} \overline{\Delta x_{n}}, \quad j=1,2, \ldots, m .
$$

where the credibility factor $z_{j n}=\frac{n a_{j}}{n a_{j}+v_{j} n}$. Then, we define the maximum premium charged to a policy holder for the $(n+1)$-th period as:

$$
\rho_{P_{n}}^{\sup }\left(\Delta X_{n+1} \mid \underline{\left.\Delta x_{n}\right)}=\sup \left\{\frac{1}{r_{n}}\left[\left(1-z_{j n}\right) \mu_{j}+z_{j n} \overline{\Delta x_{n}}\right] \mid j=1,2, \ldots, m\right\}\right.
$$

Note that if $r_{n}$ is large, the insurance company can charge a lower premium on the same risk. Again, we define the minimum premium charged to a policy holder for the $(n+1)$-th period as:

$$
\rho_{P_{n}}^{\inf }\left(\Delta X_{n+1} \mid \underline{\left.\Delta x_{n}\right)}=\inf \left\{\frac{1}{r_{n}}\left[\left(1-z_{j n}\right) \mu_{j}+z_{j n} \overline{\Delta x_{n}}\right] \mid j=1,2, \ldots, m\right\}\right.
$$

So, $\left[\rho_{P_{n}}^{\inf }\left(\Delta X_{n+1} \mid \underline{\left.\Delta x_{n}\right)}\right), \rho_{P_{n}}^{\text {sup }}\left(\Delta X_{n+1} \mid \underline{\left.\Delta x_{n}\right)}\right]\right.$ is the range of premiums that should be charged to a policy holder by an insurance company. The insurance company can decide on the amount of premium within the range $\left[\rho_{P_{n}}^{\inf }\left(\Delta X_{n+1} \mid \underline{\Delta} x_{n}\right), \rho_{P_{n}}^{\sup }\left(\Delta X_{n+1} \mid \Delta x_{n}\right)\right]$ based on the company's financial situation, and the competitors in the market. Note that both $\rho_{P_{n}}^{\mathrm{inf}}$ and $\rho_{P_{n}}^{\text {sup }}$ are non-negative in this situation.

\section{$\S 7$ Application of Bayesian risk measure to evaluate insurance risk}

In this section, we apply the Bayesian risk measure to the measurement of insurance risk. Instead of using the traditional method of ruin probability, we capture the subjective view by using the Bayesian predictive distribution. We define a risk measure which 
can be updated sequentially so that it can fit into the daily balance sheet of an insurance company. We apply the Bühlmann credibility method to obtain an approximation of the risk measure. First, we give some notations.

Suppose we have a set of manual rates (or a group of insurers) $\mathcal{T}=\left\{T_{1}, \ldots, T_{m}\right\}$. Let $x$ denote the initial surplus of an insurance company, $P_{i}$ denote the aggregate amount of premium received by the insurance company during the $i$-th period, $S_{i}$ denote the aggregate amount of claim paid by the insurance company during the $i$-th period, $I_{i}$ denote the total investment income received by the insurance company during the $i$-th period and $U_{i}$ denote the surplus of the insurance company at the end of the $i$-th period.

$$
U_{n}=x+\sum_{i=1}^{n} P_{i}-\sum_{i=1}^{n} S_{i}+\sum_{i=1}^{n} I_{i}
$$

Let $\Delta U_{i}=U_{i}-U_{i-1}, X_{j i}$ denote the amount of premium collected from the $j$-th insurer during the $i$-th period, $Y_{j i}$ denote the amount of the $j$-th insurer's claim during the $i$-th period, $N_{1 i}$ denote the number of premium payments received during the $i$-th period and $N_{2 i}$ denote the number of claims paid during the $i$-th period.

For $i=1,2, \ldots, n, n+1$, we assume the following sampling densities:

$$
\begin{array}{ll}
N_{1 i} \mid\left(\Lambda_{1}=\lambda_{1}\right) \stackrel{\text { i.i.d. }}{\sim} \operatorname{Poisson}\left(\lambda_{1}\right), & N_{2 i} \mid\left(\Lambda_{2}=\lambda_{2}\right) \stackrel{\text { i.i.d. }}{\sim} \operatorname{Poisson}\left(\lambda_{2}\right), \\
X_{j i} \mid\left(\Delta_{1}=\delta_{1}\right) \stackrel{\text { i.i.d. }}{\sim} \exp \left(1 / \delta_{1}\right), & Y_{j i} \mid\left(\Delta_{2}=\delta_{2}\right) \stackrel{\text { i.i.d. }}{\sim} \exp \left(1 / \delta_{2}\right), \\
I_{i} \mid\left(\Theta=\theta, \tau=\tau_{0}\right) \stackrel{\text { i.i.d. }}{\sim} N\left(\theta, \tau_{0}{ }^{-1}\right) . &
\end{array}
$$

We also assume the following prior densities for the unknown parameters:

$$
\begin{aligned}
& \Lambda_{1} \sim G_{a}\left(\alpha_{1}^{T_{k}}, \beta_{1}^{T_{k}}\right), \Lambda_{2} \sim G_{a}\left(\alpha_{2}^{T_{k}}, \beta_{2}^{T_{k}}\right), \Delta_{1} \sim I G\left(\gamma_{1}^{T_{k}}, \eta_{1}^{T_{k}}\right), \\
& \Delta_{2} \sim I G\left(\gamma_{2}^{T_{k}}, \eta_{2}^{T_{k}}\right), \Theta \mid\left(\tau=\tau_{0}\right) \sim N\left(\mu^{T_{k 1}} / a^{T_{k}} \tau_{0}\right), \tau \sim G_{a}\left(r^{T_{k}}, s^{T_{k}}\right),
\end{aligned}
$$

where we assume that $\Lambda_{k}$ and $\Delta_{k}$ are independent for $k=1,2$.

Note that the prior parameters can be chosen from the past manual rate $T_{k} \in \mathcal{T}$ or chosen by the insurer $T_{k} \in \mathcal{T}$ subjectively.

Let $c$ be the minimum capital requirement for an insurance company to continue its business. We define the risk measure as follows:

$$
\rho_{P_{n}}\left(\Delta U_{n+1} \mid \underline{P}_{n}, \underline{S}_{n}, \underline{I}_{n}, \min _{1 \leq i \leq n} U_{i} \geq c\right)
$$




$$
\begin{aligned}
=\sup & \left\{-E_{F_{P}^{k}}\left(P_{n+1} \mid P_{1}, \ldots, P_{n}\right)+E_{F_{S}^{k}}\left(S_{n+1} \mid S_{1}, \ldots, S_{n}\right)-E_{F_{I}^{k}}\left(I_{n+1} \mid I_{1}, \ldots, I_{n}\right)\right. \\
& \mid 1 \leq k \leq m\}
\end{aligned}
$$

Applying the Bühlmann credibility method, we have:

$$
\begin{aligned}
K^{*} & =\frac{E\left(2 \Lambda_{1} \Delta_{1}^{2}\right)}{\operatorname{Var}\left(\Lambda_{1} \Delta_{1}\right)}=\frac{2 \beta_{1}^{T_{k}}\left(\eta_{1}^{T_{k}}-2\right)}{\alpha_{1}^{T_{k}}+\eta_{1}^{T_{k}}-2} \\
z^{T_{k}} & =\frac{n}{n+K^{*}}=\text { the credibility factor } .
\end{aligned}
$$

The prior mean $=E\left(\Lambda_{1} \Delta_{1}\right)=\left(\frac{\alpha_{1}^{T_{k}}}{\beta_{1}^{T_{k}}}\right)\left(\frac{\gamma_{1}^{T_{k}}}{\eta_{1}^{T_{k}}-2}\right)=\frac{\alpha_{1}^{T_{k}} \gamma_{1}^{T_{k}}}{\beta_{1}^{T_{k}}\left(\eta_{1}^{T_{k}}-2\right)}$. So,

$$
\begin{aligned}
& E_{F_{P}^{k}}\left(P_{n+1} \mid P_{1}, \ldots, P_{n}\right) \\
\approx & \left(1-z^{T_{k}}\right)(\text { mean of prior })+z^{T_{k}} \text { (mean of observed data) } \\
= & \frac{K^{*}}{n+K^{*}}\left(\frac{\alpha_{1}^{T_{k}} \gamma_{1}^{T_{k}}}{\beta_{1}^{T_{k}}\left(\eta_{1}^{T_{k}}-2\right)}\right)+\left(\frac{n}{n+K^{*}}\right)\left(\frac{\sum_{i=1}^{n} P_{i}}{n}\right) \\
= & \frac{2 \alpha_{1}^{T_{k}} \gamma_{1}^{T_{k}}+\left(\alpha_{1}^{T_{k}}+\eta_{1}^{T_{k}}-2\right) \sum_{i=1}^{n} P_{i}}{2 \beta_{1}^{T_{k}}\left(\eta_{1}^{T_{k}}-2\right)+\left(\alpha_{1}^{T_{k}}+\eta_{1}^{T_{k}}-2\right) n}
\end{aligned}
$$

Similarly, we approximate $E_{F_{S}^{k}}\left(S_{n+1} \mid S_{1}, \ldots, S_{n}\right)$ as follows:

$$
E_{F_{S}^{k}}\left(S_{n+1} \mid S_{1}, \ldots, S_{n}\right) \approx \frac{2 \alpha_{2}^{T_{k}} \gamma_{2}^{T_{k}}+\left(\alpha_{2}^{T_{k}}+\eta_{2}^{T_{k}}-2\right) \sum_{i=1}^{n} S_{i}}{2 \beta_{2}^{T_{k}}\left(\eta_{2}^{T_{k}}-2\right)+\left(\alpha_{2}^{T_{k}}+\eta_{2}^{T_{k}}-2\right) n}
$$

Also, $E_{F_{I}}^{k}\left(I_{n+1} \mid I_{1}, \ldots, I_{n}\right)$ can be calculated exactly as follows:

$$
E_{F_{I}}^{k}\left(I_{n+1} \mid I_{1}, \ldots, I_{n}\right)=\frac{a^{T_{k}}}{a^{T_{k}}+n} \mu^{T_{k}}+\frac{n}{a^{T_{k}}+n}\left(\frac{\sum_{i=1}^{n} I_{i}}{n}\right)
$$

Therefore,

$$
\begin{aligned}
\rho_{P_{n}} & \left(\Delta U_{n+1} \mid \underline{P}_{n}, \underline{S}_{n}, \underline{I}_{n} \min _{1 \leq i \leq n} U_{i} \geq c\right) \\
\approx & \sup \left\{-\frac{2 \alpha_{1}^{T_{k}} \gamma_{1}^{T_{k}}+\left(\alpha_{1}^{T_{k}}+\eta_{1}^{T_{k}}-2\right) \sum_{i=1}^{n} P_{i}}{2 \beta_{1}^{T_{k}}\left(\eta_{1}^{T_{k}}-2\right)+\left(\alpha_{1}^{T_{k}}+\eta_{1}^{T_{k}}-2\right) n}+\frac{2 \alpha_{2}^{T_{k}} \gamma_{2}^{T_{k}}+\left(\alpha_{2}^{T_{k}}+\eta_{2}^{T_{k}}-2\right) \sum_{i=1}^{n} S_{i}}{2 \beta_{2}^{T_{k}}\left(\eta_{2}^{T_{k}}-2\right)+\left(\alpha_{2}^{T_{k}}+\eta_{2}^{T_{k}}-2\right) n}\right. \\
& \left.-\left(\frac{a^{T_{k}}}{a^{T_{k}}+n}\right) \mu^{T_{k}}-\left(\frac{n}{a^{T_{k}}+n}\right)\left(\frac{\sum_{i=1}^{n} I_{i}}{n}\right) \mid k=1,2, \ldots, m\right\}
\end{aligned}
$$

\section{Remarks:}

(1) The risk measure can be updated sequentially so that it can serve as a tool of risk measurement in the daily balance sheet of an insurance company. 
(2) Apart from viewing it as a risk measure, we can consider its' negative part as the performance index of an insurance company.

\section{$\S 8$ A modification of Bayesian risk measure for the global finan- cial market}

In this section, we give another way to capture the random effect of foreign exchange $(\mathrm{FX})$ rates in our model. A modification of the Bayesian risk measure with the random effect of FX rate included explicitly is defined, and its closed form expression can be obtained. The Bayesian risk measure is expected to give an indication of the investment risk in the global financial market. We consider a portfolio consisting of both domestic and foreign financial securities: if we invest in foreign securities, we are not only subject to risk due to the movement of the value of foreign securities, we are also subject to the risk of the movement of FX rates.

Suppose the global market in our model consists of the securities from the domestic country and $\mathrm{k}$ foreign countries. Let $m_{0}$ be the number of domestic securities and $m_{i}$ be the number of securities of the $i$-th foreign country, $i=1,2, \ldots, k$. Let the vector $\Delta Y_{r}^{(i)}$ be the changes in market values (measured in terms of the $i$-th foreign currency) per unit of all the $j$-th country's securities during the $r$-th period, i.e. $\Delta Y_{r}^{(i)}=$ $\left(\Delta Y_{1 r}^{(i)}, \Delta Y_{2 r}^{(i)}, \ldots, \Delta Y_{m_{i}, r}^{(i)}\right), i=0,1,2, \ldots, k$ and $r=1,2, \ldots, n, n+1 . f_{i t}$ denote the value of the $i$-th foreign currency at time $t$ in domestic currency, $i=1,2, \ldots, \mathrm{k}$

We would like to impose the following assumptions:

(1) The market values of the securities within each country are correlated.

(2) The market values of the securities in different countries are independent.

(3) The exchange rates are independent.

(4) The exchange rates are independent of the market values of the securities.

(5) The portfolio can be updated only at the end of each period or at the beginning of each period. Due to the presence of transaction costs and taxes in the real trading situation, this is a more realistic assumption. 
Our model is constructed as follows:

$$
\underline{Y}_{r}^{(i)}=\underline{\Theta}^{(i)} \Delta t+\underline{\varepsilon}_{r}^{(i)} \sqrt{\Delta t} ; \quad i=0,1,2, \ldots, k
$$

where $\Delta t$ is the time length of each period, $\underline{\varepsilon}_{r}^{(i)} \sim N\left(\underline{0}_{m i}, \Sigma_{i}^{-1}\right), \underline{0}_{m i}$ is a $m_{i}$-dimensional 0 vector and the $\left(m_{i} \times m_{i}\right)$-matrix $\Sigma_{i}^{-1}$ is known. The prior distribution of the random vector $\underline{\Theta}^{(i)}$ is $N\left(\underline{\mu}_{i s}, \mathcal{V}_{i s}^{-1}\right)$, where $\underline{\mu}_{i s}$ and $\mathcal{V}_{i s}^{-1}$ can be chosen by the trader $T_{s}$ subjectively. $\underline{\Delta Y}_{r}^{(i)} \mid \underline{\Theta}^{(i)}=\underline{\theta}^{i}, r=1,2, \ldots, n, n+1$, are conditionally independent and identically distributed with the common distribution:

$$
N\left(\underline{\mu}_{i s} \Delta t, \Sigma_{i}^{-1}\right) ; \quad i=0,1,2, \ldots, k
$$

After some calculations, we know that the predictive distribution of $\Delta Y_{n+1}^{(i)}$ given $\left(\underline{\Delta Y}_{1}^{(i)}, \ldots, \underline{\Delta Y}_{n}^{(i)}\right)$ is a multivariate normal distribution with mean equal to $\left(\mathcal{V}_{i s}+\right.$ $\left.n \Delta t \Sigma_{i}\right)^{-1}\left(\mathcal{V}_{i s} \underline{\mu}_{i s} \Delta t\right)+\left(\mathcal{V}_{i s}+n \Delta t \Sigma_{i}\right)^{-1}\left(n \Delta t \Sigma_{i}\right) \overline{\Delta Y}^{(i)}$, where $\overline{\Delta Y}^{(i)}=\sum_{r=1}^{n} \underline{\Delta Y_{r}^{(i)}} / n$. (Some of these calculation can be found in Siu and Yang (1998))

Let $\underline{\alpha}_{r}^{(i)}=\left(\alpha_{1 r}^{(i)}, \alpha_{2 r}^{(i)}, \ldots, \alpha_{m_{i}, r}^{(i)}\right), i=0,1,2, \ldots, \mathrm{k}$, where $\alpha_{j r}^{(i)}$ is the number of units of the $j$-th security in the $i$-th foreign country at the end of the $r$-th period (or at the beginning of the $(r+1)$-st period). If $\alpha_{j r}^{(i)}$ is equal to zero, then the $\mathrm{j}$-th security in the i-th foreign country is not included in the portfolio at the end of the $r$-th period. If $\alpha_{j r}^{(i)}$ is less than zero, then $-\alpha_{j r}^{(i)}$ is interpreted as the number of units short sold. Some countries have policies (regulations) to restrict the activity of short selling. If this is the case, we can impose some constraints on the values of $\alpha_{j r}^{(i)}$.

Let $\Delta X_{r}^{(j)}$ be the change of a portfolio in the i-th country during the r-th period in the domestic currency. Then $\Delta X_{r}^{(j)}=f_{i r} \sum_{j=1}^{m i} \alpha_{j, r-1}^{(i)} \Delta y_{j r}^{(i)}, i=1,2, \ldots, k$ and $r=1,2, \ldots, n, n+1$. Also, we assume that the process $f_{i t}$ satisfies a mean-reverting model (the Vasicek Model):

$$
d f_{i t}=a^{T_{s}}\left(b^{T_{s}}-f_{i t}\right) d t+\sigma^{T_{s}} d W_{i t}
$$

$i=1,2, \ldots, k$, where $a^{T_{s}}, b^{T_{s}}$ and $\sigma^{T_{s}}$ are non-negative constants chosen by the trader $T_{s}$ subjectively. 
Let $\mathcal{F}_{n}^{f_{i}}$ be the $\sigma$-algebra generated by the process $f_{i t}$ up to time n (i.e. $\mathcal{F}_{n}^{f_{i}}=\sigma\left\{f_{i t} \mid 0 \leq\right.$ $t \leq n\})$. After some calculations, we obtain

$$
\begin{gathered}
E_{T_{s}}\left(f_{i, n+1} \mid \mathcal{F}_{n}^{f_{i}}\right)=f_{i n} e^{-a^{T_{s}} \Delta t}+b^{T_{s}}\left(1-e^{-a^{T_{s}} \Delta t}\right) \\
\sigma_{T_{s}}\left(f_{i, n+1} \mid \mathcal{F}_{n}^{f_{i}}\right)=\frac{\sigma^{T_{s}}}{\sqrt{2 a^{T_{s}}\left(1-e^{-2 a^{T_{s}} \Delta t}\right)}}
\end{gathered}
$$

Let $\Delta X_{n+1}$ be the change of the market value (measured in the domestic currency) of a global portfolio during the $(n+1)$-th period. Then $\Delta X_{n+1}=\sum_{i=0}^{k} \Delta X_{n+1}^{(i)}$.

Let $\underline{\Delta Y^{(i)}}=\left(\underline{\Delta Y}_{1}^{(i)}, \underline{\Delta Y}_{2}^{(i)}, \ldots, \underline{Y}_{n}^{(i)} .\right)^{T}, i=0,1,2, \ldots, k$, (i.e. $\underline{\Delta Y^{(i)}}$ is a $\left(n \times m_{i}\right)$ matrix). Then, we define the risk measure as follows:

$$
\begin{aligned}
& \rho_{P_{n}}\left(\Delta X_{n+1} \mid \underline{\Delta Y}^{(0)}, \underline{\Delta Y^{(1)}}, \ldots, \underline{\Delta Y^{(k)}}, \mathcal{F}_{n}^{f_{1}}, \ldots, \mathcal{F}_{n}^{f_{k}}\right) \\
= & \frac{1}{r_{n}} \sup \left\{-E_{T_{s}}\left[\left(\underline{\alpha}_{n}^{(0)}\right)^{T} \underline{\Delta Y}_{n+1}^{(0)} \mid \Delta Y^{(0)}\right]-\sum_{i=1}^{k}\left[E_{T_{s}}\left(f_{i, n+1} \mid \mathcal{F}_{n}^{f_{i}}\right)\right.\right. \\
& \left.\left.+I\left(-E_{T_{s}}\left[\left(\underline{\alpha}_{n}^{(i)}\right)^{T} \underline{Y}_{n+1}^{(i)} \mid \Delta Y^{(i)}\right]\right) \sigma_{T_{s}}\left(f_{i, n+1} \mid \mathcal{F}_{n}^{f_{i}}\right)\right] E_{T_{s}}\left[\left(\underline{\alpha}_{n}^{(i)}\right)^{T} \Delta Y_{n+1}^{(i)} \mid \Delta Y^{(i)}\right] \mid T_{s} \in \mathcal{T}\right\}
\end{aligned}
$$

Write

$$
M_{s n}^{(i)}=\left(\underline{\alpha}_{n}^{(i)}\right)^{T}\left[\left(\mathcal{V}_{i s}+n \Delta t \Sigma_{i}\right)^{-1}\left(\mathcal{V}_{i s} \underline{\mu}_{i s} \Delta t\right)+\left(\mathcal{V}_{i s}+n \Delta t \Sigma_{i}\right)^{-1}\left(n \Delta t \Sigma_{i}\right) \underline{\Delta y}^{(i)}\right]
$$

Then,

$$
\begin{gathered}
\rho_{P_{n}}\left(\Delta X_{n+1} \mid \underline{\Delta Y^{(0)}}, \underline{\Delta Y^{(1)}}, \ldots, \underline{\Delta Y^{(k)}}, \mathcal{F}_{n}^{f_{1}}, \ldots, \mathcal{F}_{n}^{f_{k}}\right) \\
=\frac{1}{r_{n}} \sup \left\{-M_{s n}^{(0)}-\sum_{i=1}^{k}\left[f_{i, n} e^{-a^{T_{s}} \Delta t}+b^{T_{s}}\left(1-e^{-a^{T_{s}} \Delta t}\right)\right.\right. \\
\left.\left.\quad+I\left(-M_{s n}^{(i)}\right) \frac{\sigma^{T_{s}}}{\sqrt{2 a^{T_{s}}\left(1-e^{-2 a^{T_{s} \Delta t}}\right)}}\right] M_{s n}^{(i)} \mid T_{s} \in \mathcal{T}\right\}
\end{gathered}
$$

where

$$
I(x)=\left\{\begin{array}{lll}
1 & \text { if } & x>0 \\
0 & \text { if } & x \leq 0
\end{array}\right.
$$

Also, we can define the risk limits as follows:

$$
\begin{aligned}
& \rho_{P_{n}}^{\inf }\left(\Delta X_{n+1} \mid \underline{\Delta Y^{(0)}}, \underline{\Delta Y^{(1)}}, \ldots, \Delta Y^{(k)}, \mathcal{F}_{n}^{f_{1}}, \ldots, \mathcal{F}_{n}^{f_{k}}\right) \\
= & \frac{1}{r_{n}} \inf \left\{-M_{s n}^{(0)}-\sum_{i=1}^{k}\left[f_{i, n} e^{-a^{T_{s}} \Delta t}+b^{T_{s}}\left(1-e^{-a^{T_{s}} \Delta t}\right)\right.\right.
\end{aligned}
$$




$$
\left.\left.+I\left(M_{s n}^{(i)}\right) \frac{\sigma^{T_{s}}}{\sqrt{2 a^{T_{s}}\left(1-e^{-2 a^{T_{s}} \Delta t}\right)}}\right] M_{s n}^{(i)} \mid T_{s} \in \mathcal{T}\right\}
$$

and

$$
\begin{aligned}
& \rho_{P_{n}}^{\sup }\left(\Delta X_{n+1} \mid \underline{\Delta Y^{(0)}}, \underline{\Delta Y^{(1)}}, \ldots, \underline{\Delta Y^{(k)}}, \mathcal{F}_{n}^{f_{1}}, \ldots, \mathcal{F}_{n}^{f_{k}}\right) \\
= & \rho_{P_{n}}\left(\Delta X_{n+1} \mid \underline{\Delta Y}^{(0)}, \underline{\Delta Y^{(1)}}, \ldots, \underline{\Delta Y}(k), \mathcal{F}_{n}^{f_{1}}, \ldots, \mathcal{F}_{n}^{f_{k}}\right)
\end{aligned}
$$

\section{Remarks:}

In order to obtain a conservative risk measure, we multiply $M_{s n}^{(i)}$ by $\left[E_{T_{s}}\left(f_{i, n+1} \mid \mathcal{F}_{n}^{f_{i}}\right)+\right.$ $\left.\sigma_{T_{s}}\left(f_{i, n+1} \mid \mathcal{F}_{n}^{f_{i}}\right)\right]$ if $M_{s n}^{(i)}$ is negative and by $E_{T_{s}}\left(f_{i, n+1} \mid \mathcal{F}_{n}^{f_{i}}\right)$ if $M_{s n}^{(i)}$ is positive, $i=1,2, \ldots, k$. To achieve this, we introduce the indicator function $I\left(-M_{s n}^{(i)}\right)$. To define the lower risk limit, we multiply $M_{s n}^{(i)}$ by $E_{T_{s}}\left(f_{i, n+1} \mid \mathcal{F}_{n}^{f_{i}}\right)$ if $M_{s n}^{(i)}$ is positive and by $\left[E_{T_{s}}\left(f_{i, n+1} \mid \mathcal{F}_{n}^{f_{i}}\right)+\right.$ $\left.\sigma_{T_{s}}\left(f_{i, n+1} \mid \mathcal{F}_{n}^{f_{i}}\right)\right]$ if $M_{s n}^{(i)}$ is negative, $i=1,2, \ldots, k$.

\section{$\S 9$ Conclusion and Further Research}

We introduced a conditional risk measure which can be updated sequentially as more information is obtained. Then, a Bayesian risk measure was defined in order to capture the subjective view of the risk trader. For the conjugate-prior case, we obtained a closed form expression for the Bayesian risk measure. For the non-conjugate-prior case, we used the Bühlmann credibility method to approximate the Bayesian risk measure. The Bayesian risk measure can be applied to measure both financial risks and insurance risks. We have illustrated how to apply the Bayesian risk measure to calculate premiums and to measure the insurance risks. We have also given an alternative method to capture the impacts of random foreign exchange rates on global investments in our model. A modification of the Bayesian risk measure has been introduced.

For further developments of our model, different priors may be used in order to gain a more realistic model. As long as they are conjugate-prior cases, we can obtain the closed form solutions. Otherwise, we can use some simulation techniques such as the Markov Chain Monte Carlo (MCMC) method to approximate the Bayesian risk measure. The disadvantage of the MCMC method, however, is that it is time-consuming. If the data set is too large and the updating of the risk measure is frequent, the computer may not have enough capacity to handle the calculations. Another interesting problem we can 
study is the problem of the robustness of the Bayesian risk measure with respect to the change of prior distributions. This idea is similar to performing a stress test on our risk measure with respect to extreme market movements. If the risk measure is robust, then we get a narrow risk interval. Otherwise, we get a wide risk interval.

\section{References}

1. Artzner, P., F. Delbaen, J. Eber and D. Heath (1998). Coherent Measures of Risk. Working paper, Universite Louis Pasteur, France.

2. Chateauneuf, A., R. Kast and A. Lapied (1996). Choquet pricing for financial markets with frictions. Mathematical Finance, 6, 323-330.

3. Cvitanic, J. and I. Karatzas (1998). On Dynamic Measures of Risk. Working paper, Columbia University, USA.

4. Denneberg, D. (1994). Conditioning (updating) non-additive measures. Annals of Operations Research, 52, 21-42.

5. Duffie, D. and J. Pan (1997). An overview of value at risk. Journal of Derivatives, Spring, 1997, p.7 - 49.

6. Embrechts, P., C. Kluppelberg and T. Mikosch (1997). Modelling Extremal Events. Springer-Verlag.

7. Florens, J.P., M. Mouchart and J. M. Rolin (1990). Elements of Bayesian Statistics. Pure and Applied Mathematics, A Series of Monographs and Textbooks, 134. Marcel Dekker, Inc. New York and Basel.

8. Follmer, H. and P. Leukert (1998). Quantile Hedging. Working paper, Humbolt University, Berlin.

9. Holton, G. (1997). Subjective Value at Risk. Financial Engineering News, August.

10. Klugman, S.A., H.H. Panjer and G.E. Willmot (1998). Loss Models: From Data to Decisions. John Wiley \& Sons. 
11. Morgan, J.P. (1996). RiskMetrics - Technical Document. fourth edition, New York.

12. Siu, T. K. and H. Yang (1999). Bayesian value at risk, Research report, Department of Statistics and Actuarial Science, The University of Hong Kong.

13. Wang, S.S., V. R. Young and H.H. Panjer (1997). Axiomatic characterization of insurance prices. Insurance: Mathematics \& Economics, 21, 173 - 183.

14. Wang, S.S. and V. R. Young (1998). Risk-adjusted credibility premiums using distorted probabilities. Scand. Actuarial Journal, 2, 143 - 165.

15. Willmot, Gordon E. (1994). Introduction to credibility theory. Research Report, University of Waterloo.

16. Young, V.R. (1998). Families of update rules for non-additive measures: Applications in pricing risks. Insurance: Mathematics \& Economics, 23, 1 - 14. 